\title{
La représentation panique dans le théâtre de Jan Fabre
}

\author{
Amélie Mons ${ }^{1}$ (D) \\ Published online: 27 February 2019 \\ (c) The Author(s) 2019
}

\begin{abstract}
Jan Fabre's theatre was and still is a controversial one. Pretentious, elitist, too intellectual according to some, it is also seen as populist and demagogic for others. The Flemish artist divides his audience in a radical way. It is this very gap, this "incoherence" in the multiple criticisms this theatre receives that makes us think that while this form of contemporary art is maybe detested it is fore and foremost maybe not always well understood. It is indeed uneasy to apprehend a theatre that deconstructs the process of representation when the audience has been used to discussing representation and representation only. I would like to review these critiques; see how they avoid the real issues at stake, in order to show an actual lack of critical means, a lack of analytical tools, which would allow us to justly critique an innovative theatre. But most of all, I want to propose a different perspective. It is with the use of the philosophy of the French intellectual Clément Rosset, that I wish to introduce a perhaps more suitable approach. This theatre does not represent the real; it tries to be as close as possible to it. The objects on stage start to refer to themselves. In a very similar way, the concept of "représentation panique" developed by Rosset refers to a situation where a "coincidence" happens between the thing and its representation. This will help to overcome the paradox established by Jan Fabre: that of a world free from representations, yet taking place on a theatrical stage.
\end{abstract}

Keywords Contemporary theatre $\cdot$ Critical reception $\cdot$ Spectator $\cdot$ Presence $\cdot$ Real

«Les gens qui ne comprennent pas croient qu'on les provoque. Rien n'a changé depuis 25 ans que je travaille. C'est triste; la stupidité est toujours prête à surgir ». ${ }^{1}$ C'est le metteur en scène et plasticien flamand Jan Fabre qui prononça ces paroles à la fin d'un sulfureux festival d'Avignon en 2005. Son spectacle L'Histoire

\footnotetext{
${ }^{1}$ Cité par Carole Talon-Hugon (2006, p. 53), elle-même citant D. Frétard, in Le Monde (5 Juillet 2005).
}

Amélie Mons

amelie.mons@postgrad.manchester.ac.uk

1 University of Manchester, Oxford Road, Manchester M13 9PL, UK 
des Larmes, présenté dans la Cour d'Honneur du Palais des Papes avait alors reçu autant d'applaudissements que d'injures et d'incompréhensions. Très violents dans leurs critiques, les journalistes avaient entre autres dénoncé chez l'artiste une incompétence intellectuelle, un anti-intellectualisme, et avaient qualifié son spectacle de démonstration populiste. ${ }^{2}$ Le festival d'Avignon cette année aura donné lieu à une «querelle du théâtre», où chacun tenta de défendre sa propre vision du théâtre. Les deux camps s'affrontant étaient ceux d'un théâtre centré autour du texte contre ceux d'un théâtre centré autour de la figure de l'artiste, ${ }^{3}$ et donc d'un théâtre libre de faire largement appel à d'autres formes telles que la danse ou la performance, fragment de réel venant perturber la trame dramatique. Pour définir ce dernier genre, l'on pourrait de manière assez générale reprendre la définition qu'Hans-Thies Lehmann faisait du théâtre post-dramatique: «[un théâtre] davantage manifestation que signification, davantage impulsion d'énergie qu'information»(2002, p. 134). Aujourd'hui cette définition a été reprise, nuancée, précisée, mais persiste encore un conflit entre théâtre de texte et théâtre de corps; un conflit entre un théâtre «intellectuel» et un théâtre physique toujours plus proche de la danse, conflit qui n'a pourtant pas lieu d'être selon Florence Dupont (2007). A ce titre, «une révolution intellectuelle reste à faire qui change les termes du débat»écrivait-elle deux ans après le très houleux festival (2007, p. 17).

Cet article entend démontrer que les «termes du débat»dans le milieu journalistique demeurent presque inchangés, et ce, plus de dix ans plus tard. Le théâtre post-dramatique continue d'être difficilement reçu, mais surtout, mal interprété. C'est d'ailleurs ce que Jan Fabre avait déjà perçu après Avignon 2005: «Je souhaite aussi que la presse change, ait plus une fonction d'intermédiaire entre le public et le travail, qu'une fonction de destruction du travail en déclarant que c'est raté » (Le Cas Avignon, 2005). Si bien sûr cette remarque démontre le manque de crédibilité que l'artiste accorde à la presse (les journalistes n'ont pas le droit de porter d'avis négatifs), elle montre aussi que le dialogue et la compréhension entre ces artistes contemporains et le public est loin d'être facile. Longtemps habitués au théâtre de texte et à son analyse sémiologique, il paraît difficile pour la critique et le public de s'adapter en adoptant une nouvelle approche critique. «Le théâtre autiste et fière de l'être», tel que le désigne Régis Debray (2005, p. 37), ne serait ainsi pas tant autiste par choix qu'à cause plutôt d'une incompréhension toujours persistance entre l'artiste et son public. Autrement dit, si on le pense «autiste», ce n'est pas parce qu'il refuse toute communication avec ses spectateurs, mais bien parce qu'il est mal compris. Il ne s'agit alors pas tant de remettre en cause cette forme de théâtre même, mais de remettre plutôt en cause la manière dont on y assiste. C'est peut-être cela que Jan Fabre voulait exprimer dans cette phrase que l'on a citée au tout début, certes, pleine de souveraine assurance, et disons-le, teintée d'un peu de mépris.

C'est après avoir exploré cette approche critique peu satisfaisante car faussée, que l'on tentera de proposer une nouvelle approche, une nouvelle lecture du théâtre de Jan Fabre, libérée de toute tentative de déchiffrage d'un «message» que le metteur

\footnotetext{
2 Voir Talon-Hugon (2006, pp. 52-53).

3 Voir à ce compte la notion de «métaphysique de l'artiste» (Talon-Hugon 2006).
} 
en scène voudrait soi-disant adresser. Et c'est à travers un concept philosophique, celui de la «représentation panique», développé par le philosophe français Clément Rosset (2004, pp. 162-173) qu'une nouvelle ouverture semble être possible. La «représentation panique», explique-t-il, se traduit par une «coïncidence entre le réel et sa représentation». Le sujet perçoit une situation qui se représente en même temps qu'elle existe, rendant impossible pour lui l'accès à un «temps de la reconnaissance». Il n'a pas le temps de se représenter l'action intérieurement, d'où, chez Rosset, un sentiment de panique. C'est précisément cette dissociation faite entre le réel et sa signification qui permettra de comprendre comment une approche débarrassée du souci de la quête de sens est possible. En étudiant ce concept au plus près, l'on espère ici «changer les termes du débat», en proposant de nouvelles clés pour discuter le théâtre de Jan Fabre, mais aussi plus largement, une certaine esthétique du théâtre contemporain. Il s'agira en effet de centrer davantage la critique sur l'expérience intime du spectateur et de sa difficulté à appréhender ce «réel»à l'œuvre sur la scène théâtrale. Car si la scène de théâtre est censée être l'espace de représentation par excellence, la scène de Jan Fabre a étonnamment cette capacité à «révéler» le réel en venant perturber, ou plutôt complexifier, les habitudes de perception de ses spectateurs.

Aujourd'hui une partie du théâtre contemporain (et d'une certaine mesure de l'art contemporain en général) est approchée d'une manière très théorique; à l'entrée d'une exposition est délivré un large feuillet expliquant ce que l'artiste «a voulu dire»avec telle installation; au théâtre il aura fallu comprendre le «projet»de l'artiste pour pouvoir pleinement apprécier son travail... L'approche critique journalistique du théâtre contemporain n'est pas en reste, ayant trop souvent comme ligne d'horizon principale une recherche quasi systématique de sens. Pourtant, force est de constater que cette approche n'est pas toujours satisfaisante eu égard à certains artistes. Ainsi, les spectacles de Jan Fabre, mais aussi d'autres, ${ }^{4}$ souffrent d'une absence d'intérêt porté pour tout ce qui ne touche pas le «sens», le «message», la «signification» de leurs spectacles. Une mise en garde toutefois est nécessaire: ces spectacles sont bel et bien remplis de symboles, de références et de sens dramatique; mais là n'est pas toujours, il faut bien l'admettre, leur but premier. Nombre de critiques journalistiques tendent à automatiquement dévaluer un spectacle dont le sens serait demeuré obscur, se souciant guère de «l'impact»qu'il aurait pu avoir sur le public. Or ces spectacles contiennent souvent de la performance artistique. Chez Jan Fabre, elle se traduit par une esthétique de l'épuisement de l'acteur, à travers la répétition d'un geste ou d'une action, ou à travers une danse particulièrement physique. Ces moments-là, si centraux dans les spectacles, sont rarement discutés. Mais c'est aussi le public lui-même qui se montre réticent aux spectacles incluant de la performance et s'éloignant de la trame dramatique. La réception est brouillée parce que le message transmis n'est plus clair. En incluant du «réel» sur scène, l'artiste prend le risque de couper la communication avec une grande partie de la salle parce qu'il ne répond pas à l'attente de nombreux spectateurs; ce qu'il propose n'est pas à consommer, à interpréter. Pour Fabre, le spectateur n'est pas perçu comme un destinataire

\footnotetext{
${ }^{4}$ Que l'on pense au théâtre de l'italien Romeo Castellucci ou de l'argentin Rodrigo Garcia par exemple.
} 
qui déchiffrerait le message du metteur en scène mais comme un récepteur libre de percevoir. $^{5}$

Né à Anvers en 1958, Fabre fut d'abord plasticien avant de se lancer dans les arts de la scène dès la fin des années 1970. C'est véritablement en 1982, avec C'est du théâtre comme il était à espérer et à prévoir qu'il est reconnu comme metteur en scène. Ses spectacles mêlant danse, peinture et chant sont quasiment tous marqués par cette esthétique de la performance. Ses acteurs-souvent issus des milieux de la danse, sont nommés, après quelques années de formation et de travail commun, «guerriers de la beauté». Ces guerriers mettent leur corps au service de l'art de leur metteur en scène, quittes à en souffrir physiquement. Au cours du spectacle (ou de l'entraînement) les acteurs devront parvenir à passer du stade de l' «act»au stade de l' «acting». A travers la répétition d'un mouvement, d'un geste ou d'une action, ils finiront par abandonner toute trace d'interprétation pour n'être plus que dans la «vérité du moment». A ce titre, Mount Olympus, joué en 2015, est un spectacle de vingt-quatre heures, empreint de ces répétitions, qui demanda presque autant d'efforts à ces vingt-sept acteurs qu'à ses spectateurs. Ces spectacles contenant également souvent de la nudité, étant parfois sexuellement explicites, ou simplement violents, font loin de faire l'unanimité, et un nouveau spectacle est souvent synonyme de nouvelle polémique, même si aujourd'hui le nom de Jan Fabre est célèbre et reconnu.

On l'aura compris, la forme chez Jan Fabre (entendue comme le travail portant sur les corps), est primordiale. Pourtant, en analysant ses spectacles, la critique française a fortement tendance à opposer, de manière assez réductrice, la forme $\mathrm{au}$ fond (compris comme le «propos»du metteur en scène). Concernant Histoire des Larmes présenté à Avignon en 2005, Rosita Boisseau concluait dans Le Monde: «La beauté plastique ne masque pas le déficit de sens» (9 Juillet 2005). L'idée d'opposer l'esthétisme au sens, la forme au fond, permet toujours de placer ce dernier au rang supérieur. Si la forme est intéressante mais le fond ne suit pas, alors juge-t-on que c'est raté. Toujours dans Le Monde, Fabienne Darge et Brigitte Salino qualifiaient plusieurs spectacles d'Avignon comme «faibles sur le plan artistique [...] sans méditation et sans véritable réflexion» (12 Juillet 2005). Quant à l'Orgie de la Tolérance, spectacle présenté en 2009, Fabienne Pascaud, critique de théâtre pour Télérama concluait sa revue très négative par: «On ne sait plus ce qu'il veut raconter». Très récemment, Emmanuelle Bouchez, pour le même journal, écrivait quant au spectacle The Sea Within mis en scène par Lisbeth Gruwez (formée par Jan Fabre) que «l'esthétique formelle» finissait par «lasse[r]» (13 Juin 2018). Selon Carole Talon-Hugon, trop de critiques voient encore dans le théâtre de la performance une manière de «cacher son vide sous le renouvellement des formes» (Talon-Hugon 2006). Il est étonnant que l'absence de sens soit nécessairement perçue comme du vide, ou du moins comme quelque chose de rédhibitoire. Cela ne reviendrait-il pas à considérer tout ce qui ne touche pas à l'interprétation intellectuelle comme étant indigne d'intérêt? Ce refus de

\footnotetext{
5 A ce sujet, voir le concept de «cinquième mur»dans Le théâtre et ses publics: la création partagée (2012).
} 
considérer les formes sans un fond qui leur serait attaché est au centre de la critique de Prometheus Landscape II, publiée par Raphaël de Gubernatis pour Le Nouvel Obs (4 Avril 2011):

Prometheus Landscape II, sa dernière invention que s'empresse de montrer le Théâtre de la Ville comme s'il s'agissait d'une urgence absolue, comme s'il y allait de notre compréhension du monde. Quoi en effet de plus pertinent pour mieux saisir et le mythe et le monde qu'un homme à genoux se masturbant curieusement au pied de Prométhée enchaîné ? Qu'un gros lard sodomisant un éphèbe ou qu'une femme fessée dévêtue pour évoquer le titan condamné par Zeus pour avoir volé le feu et l'avoir donné aux hommes?

Est ici dénoncée une trivialité poussée à l'extrême, qui, assume le journaliste, aurait pour but de mieux faire comprendre à son spectateur le rapport des hommes au monde. Mais qui prétend cela? Certainement pas Jan Fabre. Ou du moins, ce n'est pas à travers certains thèmes sur scène tels que la masturbation que le public aura la chance d'élargir son rapport au monde, comme semble Raphaël de Gubernatis l'insinuer. Il s'agit davantage de voir comment ces thèmes sont traités, la manière dont ils sont insérés et présentés sur une scène de théâtre, pour ne pas uniquement s'arrêter à la réalité simple de l'action. C'est le problème récurrent chez les critiques de théâtre commentant Jan Fabre aujourd'hui: arrêter tout commentaire dès lors qu'il s'agit de la forme. Sauf que le problème avec Fabre, c'est que son travail touche essentiellement à la forme. Comme si la forme elle-même était indigne d'être commentée, le journaliste continue: «Ce qui est réjouissant, chez Jan Fabre, et au fond de bien confortable pour l'observateur, c'est, quel que soit le sujet qu'il prétende aborder, qu'on y trouvera des scènes de masturbation, de sodomie, des acteurs en compissant d'autres, des femmes et des hommes montrant leur anatomie sous un angle de préférence dégradant» (4 Avril 2011). C'est fortement réduire son théâtre que de s'arrêter au traitement cru du corps sans tenter de comprendre non pas en quoi cela sert le propos du spectacle (car l'on n'y trouverait en effet rien à dire), mais en quoi cela sert à déstabiliser et complexifier la perception du réel (et donc de la forme) pour le spectateur. En réalité, Hans-Thies Lehmann envisageait lui-même déjà cette même opposition réductrice entre le sens et la forme en parlant du théâtre post-dramatique (2002, p. 154):

Lorsque les signes n'offrent plus de synthèse, mais pourtant encore, des références à un 'contenu', ils demeurent quand même assimilables dans un travail associatif labyrinthique. Mais lorsque ces références ne fonctionnent plus du tout, la réception se retrouve face à un refus encore plus radical: la confrontation avec l'immédiateté 'muette' et dense des corps, des matières et des formes. Le signifiant ne fait plus que se communiquer soi-même, ou plus précisément, sa présence. La perception se trouve reléguée à une perception purement structurelle.

Puis parlant plus précisément du travail de Jan Fabre:

Les acteurs, les éclairages, les danseurs etc., s'offrent à une observation purement formelle, le regard ne trouve aucune occasion de dégager une significa- 
tion symboliste au-delà le réel, mais est immobilisé à la "surface" dans son activité visuelle, avec délectation ou ennui, selon les cas.

C'est ainsi qu'est largement perçu le théâtre de Jan Fabre; une forme particulière dont le fond fait souvent défaut. Certains adhèrent à cette esthétique, d'autres non. Par conséquent, le théâtre de Jan Fabre est souvent dénoncé comme «vulgaire » (Cena, 8 Juin 2018), et l'artiste se voit régulièrement qualifié d'imposteur (De Rycke, 23 Juin 2017). L'on souhaiterait ici réfuter cette approche, qui semble être celle d'un théâtre construit autour d'un texte, non celle d'un théâtre comme celui de l'artiste flamand. L'on tend à refuser l'idée que lorsque la signification disparaît, le spectateur n'aurait plus d'autre possibilité que de sombrer dans une «observation purement formelle», aussi repoussante et scandaleuse que certains puissent la juger. En fait, au lieu de dévaluer constamment la forme, c'est peut-être la forme ellemême qu'il faudrait explorer pour y déceler une nouvelle forme de sens. Le concept rosséen de «représentation panique» nous apprend une chose: cette «immédiateté muette et dense des corps » a autre chose à offrir à son spectateur qu' une simple observation formelle. Elle a à offrir une nouvelle approche du réel.

Un automobiliste est suivi à la trace par un camion-citerne. Troublé par cette situation qui a priori n'a aucun sens, il tente à plusieurs reprises de lui échapper en s'arrêtant, en le laissant passer, en accélérant, ou en changeant de direction à la dernière minute. Mais rien n'y fait, la vue angoissante du camion revient inlassablement dans son rétroviseur. Cette étrange poursuite fait l'objet du film Duel, réalisé par Steven Spielberg et sorti en 1971. Elle est aussi l'exemple que le philosophe Clément Rosset choisit pour développer son concept de «représentation panique » dans l'essai Le Réel, Traité de l'Idiotie (2004, pp.162-173). La «représentation panique», écrit-t-il, se traduit par une «coïncidence entre le réel et sa représentation». Le sujet perçoit la situation et sa représentation dans le même temps, lui rendant impossible l'accès à un «temps de la reconnaissance». L'automobiliste est ainsi heurté par un réel sur lequel il n'a pas de prises. Il n'a pas le temps de se le représenter intérieurement (grossièrement ici, de comprendre la situation), d'où, chez Rosset, un sentiment de panique. Attention toutefois, cette expérience du réel n'est en rien soudaine (comme le terme «panique»pourrait le laisser présupposer). Elle se déroule sur l'intégralité d'une journée, et c'est précisément cette durée qui rend la situation de plus en plus incompréhensible et inassimilable, puisqu'une éventuelle explication perd progressivement sa pertinence. Ce concept nous invite à revoir notre approche du réel, et plus précisément, l'idée même de sa perception. D'un des différents sens du latin percipiō qui signifie «saisir», «s'emparer de», «envahir», la perception garde aujourd'hui une connotation personnelle et subjective: percevoir, c'est voir pour moi, à partir de moi. En ce sens, un sujet qui perçoit, est un sujet qui s'empare du réel, l'envahit, et de ce fait, le transforme. En s'appropriant le réel, en se le représentant intérieurement, il projette sur lui tout un imaginaire, toute une batterie de concept, tout un historique d'expériences: il perçoit car il est capable de reconnaître, de voir en le réel ce qu'il connaît déjà, d'assimiler le réel à l'aune de ses propres représentations. C'est ce que le protagoniste de Duel est incapable de faire parce que cette course-poursuite se présente in medias res, sans avertissement ou explication. Le fait qu'il soit poursuivi par un camion n'a pas de sens dans le film; 
où plutôt il n'y a pas de sens à chercher. C'est comme ça et c'est tout nous dit Rosset. Le philosophe fait ainsi parler le protagoniste: «Au fond, c'est tout simple, il y a un camionneur qui essaie depuis ce matin de me tuer-voilà, c'est comme cela, c'est tout, c'est incroyable mais cela est et il n'y a rien à chercher d'autre » (2004, p. 168). Pour Rosset, il n'y a aucune signification à chercher qui pourrait expliquer la raison de l'action. La chose va donc exister en même temps que sa représentation parce que va néanmoins subsister un certain sens de la mise en scène perçue par le protagoniste. En effet, le thème de la course-poursuite en voiture est un thème rodé, bien connu dans l'imaginaire collectif. Notre héros ne comprend donc pas pourquoi il est poursuivi, le sens de cet événement mais quelque chose dans cette poursuite ne lui est pas tout à fait inconnu; il en devine non le sens mais la fatalité («c'est comme cela, c'est tout, c'est incroyable mais cela est»). Il y a en somme «reconnaissance de quelque chose qui n'a pas encore été connu»(2004, p. 167). Il ne comprend pas pourquoi le camion le poursuit mais il comprend d'instinct qu'il continuera à le poursuivre: il y a en ce sens, non pas une compréhension intellectuelle mais une certaine compréhension intuitive de la chose, difficilement cernable.

« Tout le mystère du film», explique Rosset, «réside dans cette coïncidence par laquelle la chose arrive et se représente dans le même temps, et non dans l'identité du camionneur ou la nature de ses mobiles» (2004, p. 168). Pour reprendre une de ses formules, il n'y a pas de mystère «dans » les choses, mais un mystère «des » choses (2004, p. 47). Et c'est à ce mystère que notre automobiliste est confronté: non pas le mystère du pourquoi de la chose (et l'on voit ici à quel point Clément Rosset se détache de la tradition philosophique), mais le mystère de la chose elle-même comme lui étant présente. Un mystère simple en somme, libre de toute hypothèse ou spéculation; un mystère premier, «à la lisière de [1'] existence » de la chose $(2004, \mathrm{p}$. 47). D'où justement le sentiment de panique du protagoniste, qui se trouve confronté à un mystère qui échappe à la prise intellectuelle (et rassurante) du «pourquoi » : la question du pourquoi ne pouvant trouver d'explications plausibles, le temps de la délibération échoue. En fait, là où le réel est ordinairement assimilé grâce à un système de représentations (ce qui lui permet de faire «sens»), la scène de coursepoursuite ne peut l'être parce qu'elle et sa représentation arrivent d'un bloc. Ainsi est-elle sans «double»; le héros pressent qu' elle est inévitable, justement parce qu'il perçoit cette représentation, cette forme de mise en scène immédiate du réel. Et c'est bien ce qui rend l'événement, dans ce cas-ci, si terrifiant.

Cet exemple d'un sujet qui percevrait le réel de manière immédiate, sans le filtre d'une représentation et qui pourtant, continuerait à voir dans ce réel un certain sens de la mise en scène (mais dont il ne serait étrangement pas responsable) semble de bien des manières faire écho au spectateur assistant à un spectacle de Jan Fabre. En effet, parce qu'il inclut volontiers de la performance artistique dans ces spectacles, Jan Fabre propose une action «réelle»dans un cadre de représentation. Fabre demande à ses acteurs des prestations particulièrement physiques, dont l'épuisement des corps -plus ou moins intense selon les spectacles-, est réel. Ce à quoi peut assister le spectateur est donc bien une bride du réel, une action prenant place dans le temps de l'ici et maintenant. Et pourtant, l'artiste se produit dans des salles très souvent frontales avec un cadre de scène (Théâtre de la Ville, Centres Nationaux Dramatiques...); en bref des salles qui proposent traditionnellement une scène 
comme espace de l'illusion, ou du moins espace de la fiction. Est ainsi montrée, dans un espace de la représentation, un espace signifiant, une action, qui elle, à tout à voir avec le réel. Autrement dit, l'on assiste à l'émergence d'un nouveau rapport au spectacle (un rapport qui pourrait paraître «accidentel» mais qui est en réalité bien souvent maîtrisé comme nous le verrons), où le temps réel semble se retrouver mis en scène «malgré lui ». Ainsi le théâtre de Jan Fabre et le concept de «représentation panique » peuvent être mis en parallèle: il s'agit pour le sujet (l'automobiliste ou le spectateur) de percevoir une action immédiate, prenant place hic et nunc mais conservant un sens de la mise en scène; ce sens intervenant exactement au même moment que l'action elle-même, et n'étant pas, comme c'est presque toujours le cas dans la perception humaine, préétabli. En fait, le spectateur ne peut «comprendre»le sens de l'action car rien ne l'explique (rien ne l'insère dans la trame dramatique ou dans un système signifiant construit par le spectacle); il ne peut que constater que le sens de l'action est inhérent à l'action elle-même. Rosset disait à propos de la «représentation panique»: «il s'agit d'une sorte de signal vide, de signalant sans signifié [...] le message y est vide et l'émission sans émetteur» (2004, p. 167). $\mathrm{Au}$ spectateur qui peut-être pensait pouvoir envahir tranquillement la scène de ses arrières-mondes, il réalise soudain, avec effroi ou joie selon les cas, que la chose perçue ne peut être assimilée sur le mode de la représentation. La performance sur scène permet de faire entrevoir un réel sans double parce qu'elle met justement en avant la distorsion entre réel et représentation. Ainsi le réel sur scène peut être plus frappant que le réel dans la vie de tous les jours parce qu'il intervient à un moment où il n'était pas attendu, et révèle, par le contraste de la scène, l'échec de notre habitude à tout nous représenter.

Voyons maintenant comment ce concept pourrait aider à développer une nouvelle méthode. Un des exemples les plus révélateurs se trouve dans Histoire des Larmes, présenté à Avignon en 2005, dans la Cour d'Honneur du Palais des Papes. Les seize premières minutes du spectacle (sur un total d'une heure quarante et une) montrent une dizaine de femmes disposées sur l'ensemble de la scène, toutes vêtues de blanc, allongées par terre sur le dos, la tête posée sur des oreillers. Elles hurlent d'une voie très aigue et pleurent à la manière des nourrissons, s'agitant dans tous les sens. Une harpiste, au centre de la scène, joue une musique douce mais que l'on n'entend à peine tant les cris sont stridents. Cette scène se présente in medias res, sans point d'accroche possible pour le spectateur qui pourrait l'aider à dégager une signification. Aucune explication n'est donnée: le spectateur ne sait pas pourquoi cela se passe ainsi, la raison de cette scène si longue et pourtant il sent après quelques minutes que cela va durer; que le sens est contenu dans la présence et la durée de l'action elle-même. Comme la représentation panique, il va ainsi non pas comprendre le pourquoi de la situation mais va davantage avoir une compréhension du caractère inévitable de cette même action, car si l'action ne fait pas sens, elle est pourtant mise en scène. De même, les acteurs semblent ici être moins dans l'interprétation d'un personnage que dans la performance, aucune marque de l'individu, aucun style de jeu ne se démarquent. La scène semble être sans intention, sans émetteur: on ne sait pas d'où viennent ces jeunes femmes qui se comportent ainsi et l'on doute soudain que la scène est en fait quoi que soit à nous dire. Elle se présente plutôt comme une énigme qui demeurerait nécessairement sans réponse. Rappelons ce que disait 
Clément Rosset sur la représentation panique: «La chose y arrive en même temps que ses signaux, l'une et les autres se confondent chronologiquement et logiquement. C'est pourquoi le message y est vide et l'émission comme sans émetteur» (2004, p. 167). Le spectateur, désorienté, pourra être irrité de cette scène d'ouverture qui ne lui laisse pas le temps (ou plutôt les clés) pour déchiffrer ce qui demeure être, à ce stade, un mystère. Bien sûr, s'il le veut absolument, il pourra toujours y dégager un sens, notamment a posteriori. En effet, «là où il y a un chemin, on peut toujours trouver une volonté»assure Rosset (2004, p. 11). Mais force est de constater qu'au moment précis où la scène est perçue, elle est inassimilable, et s'il choisit de ne pas rejeter la scène sous prétexte que «c'est n'importe quoi », alors il peut pénétrer un temps autre; un temps où le réel le frappe de plein fouet, lui retire son statut de sujet souverain pour lui révéler une réalité crue, sans fard.

L'on voit déjà dans cet exemple se dessiner un thème crucial dans l'œuvre de Jan Fabre: la durée de la répétition. C'est par la longue répétition, notamment de l'effort physique, qu'il entend déstabiliser la perception de son spectateur, ou du moins, obliger ce dernier à modifier sa perception pour finalement ne plus être capable de projeter des «significations imaginaires » (2004, p. 7). En effet, c'est par l'épuisement du signe que la scène parviendra à atteindre ce «vide» dont il était question plus tôt, mais qui ici, est si important. Plus l'action physique durera, plus le spectateur sera perturbé par la «collision» entre le caractère réel de l'action et le cadre de scène. A travers la répétition, Jan Fabre tend à nous faire revoir nos premières impressions: si l'on pensait comprendre cette action comme une protestation contre l'ordre établi par exemple, alors l'on se sentira vite un peu bête, parce que la répétition incessante nous obligera à revoir cette hypothèse et nous montrera à quel point elle était subjective et autocentrée. La représentation de la chose deviendra de plus en plus obscure jusqu'à qu'elle se fonde dans la chose elle-même: la chose et ses signaux se confondront, la chose et sa représentation coïncideront. C'est prendre mesure que le réel, pour reprendre une formule de Clément Rosset, «est riche de quelque chose à quoi aucun savoir ne peut préparer, qu'aucune représentation ne peut figurer à l'avance: précisément sa qualité d'être réelle, le mystère de sa présence» (2004, p. 164). La répétition chez Fabre est souvent physiquement intense, les acteurs devant répéter une action jusqu'à ce que leur corps ne puisse tout simplement plus le supporter. La première fois que le spectateur percevra l'action sera ainsi bien différente de la dernière. Si la première est perçue comme une représentation d'une chose, la dernière est perçue comme la chose elle-même. C'est précisément ce processus que Fabre désigne par la formule «From Act to Acting». Il semble ainsi dire à son spectateur une chose qui parait bien simple: ce que vous voyez n'est rien d'autre que ce qui se fait. ${ }^{6}$ Etant lui-même spectateur assidu des spectacles de Jan Fabre, le chercheur Luk Van Den Dries explique quant à l'expérience du public (2005, pp. 21-22):

\footnotetext{
${ }^{6}$ Expression employée par Olivier Dubouclez à propos de la philosophie de Clément Rosset lors d'une journée d'études organisée à Liège le 27 avril 2018 et intitulée «Ethique et Ontologie autour de Clément Rosset».
} 
La répétition est la stratégie la plus importante dans l'œuvre de Jan Fabre. D'innombrables scènes se craquellent sous son poids [...] Nombreuses sont les scènes et les pièces de Fabre où l'on assiste à cette répétition exacerbée, qui n'atteint pas seulement l'acteur mais aussi le spectateur, car le regarder épuise tout autant. En répétant toujours ces gestes minimalistes, le spectateur est invité à revoir sa première impression. Les détails prennent toute leur importance, surtout quand la répétition est conduite à l'unisson par le groupe. C'est alors qu'elle change la façon même de regarder. L'épuisement suscite sa propre dimension du temps et l'on se met à regarder différemment, à un autre niveau de perception. Une sorte d'ivresse s'empare alors du spectateur. Mais le corps et le regard ne sont pas les seuls à se lasser, la signification s'épuise, elle aussi.

Par exemple, dans Le Pouvoir des Folies Théâtrales, présenté en 1984 et repris en 2012, une actrice se trouvant en face du premier rang des spectateurs tente désespérément de monter sur scène, mais un des acteurs y étant déjà présent l'en empêche. A chaque fois qu' elle essayera de remonter, il la repoussera violemment. Cette scène dure environ vingt minutes. Le visage de l'actrice devient de plus en plus rouge, elle transpire et respire de plus en plus fort, l'effort s'intensifiant. Là où le spectateur aurait pu percevoir tout un symbole de répression, un signe méta-théâtral etc., il se rend compte petit à petit que ce à quoi il assiste n'est ni plus ni moins qu'un effort physique de plus en plus intense de la part d'une femme continuellement repoussée par un homme. Dans Je Suis Sang, présenté à Avignon en 2003, une scène plutôt marquante joue également du processus de la répétition. La scène se déroule ainsi: plusieurs personnages habillés comme des mariées, répartis sur l'ensemble de la scène, ressentent le besoin de couper une partie de leur corps; des personnages vêtus comme des chevaliers arrivent de l'arrière-scène pour couper avec leur épée la partie du corps que les mariées leur présentent, les laissent se vider de leur sang puis les portent jusqu'à l'arrière-scène, d'où ils proviennent. Ils les posent alors sur le sol. Doucement, elles se réveillent et commencent à lécher leur sang qui a laissé une trainée sur le sol. Elles finissent par revenir à leur place initiale, et ayant regagné leurs forces, elles demandent à nouveau qu'une partie de leur corps soit sectionnée. Cette scène se répètent indéfiniment jusqu'à ce que les acteurs jouant les chevaliers, épuisés de porter ces mariées, ne puissent tout simplement plus être dans une forme d'interprétation, aussi fine soit-elle, et ne parviennent plus qu'à rassembler leurs dernières forces sur la tâche à accomplir_-déplacer ces femmes. Là où encore une fois l'on pouvait penser assister à une scène de pietà, on se rend rapidement compte qu'il ne s'agit en somme que d'hommes, épuisés, portant des femmes, et tentant de «tenir» le plus longtemps possible. Luk Van Den Dries témoigne: «L'image se lézarde, les muscles se mettent à trembler, la sueur perle sur les fronts et le temps concret prend sa revanche sur les corps épuisés. Les amantes sont plus traînées que portées, et finissent par s'effondrer sur le sol»(2005, p. 21). Cette scène est en réalité un exercice que Fabre donne à ses acteurs pour leur permettre, encore une fois, de comprendre la différence entre «act» et «acting». Luk Van Dries aura cette formule éclairante à propos d'un autre spectacle de Fabre, C'est du théâtre comme c'était à espérer et prévoir: «Le son de son corps qui s'écrase sur un plancher en bois n'est ni plus ni moins celui d'un corps quelconque qui s'écrase sur un plancher 
en bois» (2005, p. 19). Ce qui se passe sur scène finit par être perçu comme ce qui se passe sur scène et rien d'autre, comme un réel sans représentation, dont pourtant l'accès est si loin d'être évident.

Cette approche si crue, voire cruelle, d'un corps sans artifice, est également à comprendre à la lumière de la fascination du metteur en scène pour la biologie. Fabre s'est toujours montré très intéressé par tout ce qui touchait le corps en tant qu'organe vivant. Nombreux sont ses spectacles qui présentent des scènes de dissection (Requiem for a Metamorphosis, Je Suis Sang...). Cela engendre d'ailleurs un reproche qui lui est très souvent adressé: celui de proposer un théâtre «obscène». Sang, urine, nudité, actes sexuels... Ce qui se joue sur scène peut facilement être vu comme trivial et une fois de plus indigne d'intérêt. Fabre ne joue jamais d'effets de distanciation comme c'est le cas pour d'autres artistes contemporains. Au contraire, ces acteurs sont corps entiers jetés dans la bataille. Fabre se vante d'ailleurs de travailler comme un biologiste, de proposer dans ces spectacles une étude des corps et de ses mécanismes. Il veut montrer comment les joints s'articulent, comment la douleur affecte le contrôle du corps... L'individualité de l'acteur disparaît dans ce théâtre: ce que l'on voit souvent, ce sont des corps et juste des corps. ${ }^{7}$ Etymologiquement, obscène vient du latin ob-scenus, signifiant ce qui est devant la scène, autrement dit, en dehors de la scène. C'est ce qui est indigne d'être regardé, ce qui doit rester caché. Pourtant, c'est justement ce qui est là avant toute chose, ce qui est là avant même la scène. L'obscénité sur scène est dérangeante car elle impose un rapport trop direct avec ce qui s'y joue, un rapport trop «primitif» au corps. Elle montre un corps, en fait, débarrassé de toutes représentations, et qui en toute logique, ne devrait donc pas se trouver sur une scène de théâtre. C'est encore une fois ce décalage entre espace de représentation et action qui s'y joue qui vient déstabiliser la perception du spectateur, en venant lui proposer une approche bien plus directe du réel, aussi effrayante -et repoussante pour certains, soit-elle. La vue du corps, montré dans toute son organicité, avec ses fluides, constitue ainsi une double transgression: non seulement il est présenté à la vue des autres (là où on devrait le dissimuler), mais il est en plus présenté sur scène, l'espace d'observation par excellence. Cette «réalité première » du corps organique reste ainsi mise en scène, comme dans le cadre de la représentation panique. Et c'est bien là ce qui cause le malaise des spectateurs dont l'indignation témoigne peut-être parfois d'une difficulté à appréhender un réel sans représentations.

Le théâtre de Jan Fabre n'est pas toujours abordé de manière pertinente. Sans non plus vouloir, comme le metteur en scène, ôter aux commentateurs tout droit au sens critique-qu'ils soient le public en général ou les médias, il convient d'adopter de nouvelles propositions, de nouvelles clés, pour aborder un tel art de la mise en scène. $S$ 'interroger sur la manière dont la scène challenge la perception

\footnotetext{
7 Sa méthode de formation d'acteurs a d'ailleurs récemment fait réagir dans le cadre du débat\#MeToo. Certains de ses acteurs (majoritairement des femmes) l'ont accusé de les avoir attaqués sur leur physique et de les humilier pendant les répétitions. Ils ont aussi dénoncé un manque d'humanité et l'impression de se faire traiter en objets par Fabre et sa compagnie Troubleyn. La lettre ouverte écrite par les victimes peut se trouver à cette adresse: https://www.rektoverso.be/artikel/open-letter-metoo-and-troubleynjanfabre. L'article a été écrit antérieurement à cette affaire.
} 
du spectateur à l'aune du modèle de la représentation panique en a été un exemple. Il aura au moins permis de couper court à la fameuse critique «ça n'a aucun sens », ou «c'est gratuit» puisque qu'il permet justement de valoriser cette fuite du sens; de le rendre enfin digne d'intérêt. A travers la performance, le théâtre fabrien demande quelque chose d'ambitieux à son spectateur: appréhender le réel sans représentations, et sur une scène en plus! Il demande ce que Rosset demandait déjà dès les années 1970, à savoir un retour à un rapport au monde plus essentiel, plus direct, débarrassé d'interprétations intellectuelles superflues. Paradoxalement, c'est avec la scène que Fabre a trouvé un moyen de rendre compte de cette urgence. Au lieu de chercher à décortiquer ses spectacles, les signes sur la scène, et ce qu'il a bien voulu dire, pourquoi ne pas ainsi simplement respecter le mystère qui s'en dégage? Car c'est justement en s'interrogeant sur le réel sur scène, sans jamais le dénaturer à coups d'interprétations et de projections de «significations imaginaires », qu'un mystère apparaît; un mystère qui n'a pas été construit par l'homme, un mystère de la réalité simple en somme, «un mystère des choses » et non «dans les choses » (Rosset 2004, p. 47). Cet article a voulu démontrer que c'est à travers une nouvelle méthodologie qu'il pourrait être appréhendé. Et s'il est vrai que l'artiste peut en effet se montrer quelquefois prétentieux et suffisant, il faudra savoir tirer le meilleur d'un théâtre qui met en œuvre un rapport au réel simple, primitif, mais pourtant si étrange, dont nous aurions souvent besoin.

OpenAccess This article is distributed under the terms of the Creative Commons Attribution 4.0 International License (http://creativecommons.org/licenses/by/4.0/), which permits unrestricted use, distribution, and reproduction in any medium, provided you give appropriate credit to the original author(s) and the source, provide a link to the Creative Commons license, and indicate if changes were made.

\section{Réfèrences}

Banu, G., \& Tackels, B. (dir.) (2005). Le cas Avignon 2005: regards critiques. Paris: L'entretemps.

Boisseau, R. (9 Juillet 2005). Le corps selon Jan Fabre laisse Avignon perplexe. Document en ligne. Le Monde. https://www.lemonde.fr/culture/article/2005/07/09/1-histoire-des-larmes-le-corpsselon-jan-fabre-laisse-avignonperplexe_671258_3246.html. Visité le 20/07/2018.

Bouchez, E. (2018). The sea within. Télérama. Ed., 3570, 75.

Cena, O. (8 Juin 2018). Pénis en érection, vierges ridiculisées... Jan Fabre sombre dans la vulgarité. Document en ligne. Télérama. https://www.telerama.fr/sortir/penis-en-erection,-vierges-ridic ulisees-jan-fabre-sombre-dans-la-vulgarite,n5676894.php. Visité le 20/07/2018.

Darge, F., \& Salino, B. (12 Juillet 2010). 2005, l'année de toutes les polémiques, l'année de tous les paradoxes. Document en ligne. Le Monde. https://www.lemonde.fr/culture/article/2005/07/27/ avignon-2005-1-annee-detoutes-les-polemiques-et-de-tous-les-paradoxes_675755_3246.html. Visité le 20/07/2014.

De Gubernatis, R. (4 Avril 2011). Jan Fabre, le Prométhée des esprits indigents ? Document en ligne. Le Nouvel Obs. https://www.nouvelobs.com/culture/20110404.OBS0727/jan-fabre-le-prome thee-des-esprits-indigents.html. Visité le 20/07/2018.

De Rycke, J.-P. (23 Juin 2017). Contre-manifeste de l'art contemporain: pour un ré-enchantement de la création. Document en ligne. Le Figaro. http://www.lefigaro.fr/vox/culture/2017/06/23/31006 -20170623ARTFIG00366-contre-manifeste-de-1-art-contemporain-pour-un-re-enchantement-dela-creation.php. Visité le 20/07/2018. 
Debray, R. (2005). Sur le pont d'Avignon. Paris: Flammarion.

Delhalle, N. (dir.) (2012). Le théâtre et ses publics: la création partagée. Colloque de Liège. Paris: Les solitaires intempestifs. Le programme de la conférence est consultable en ligne. https ://f-origin.hypotheses.org/wp-content/blogs.dir/33/files/2012/09/COLLOQUE-PROSPERO_ FR.pdf. Visité le 09/01/2019.

Dupont, F. (2007). Aristote ou le vampire du théâtre occidental. Paris: Flammarion.

Lehmann, H.-T. (2002). Le théâtre postdramatique. Paris: L'Arche.

Rosset, C. (2004). Le Réel, Traité de l'idiotie. Paris: Les Editions de Minuit.

Talon-Hugon, C. (Juin 2006). Avignon 2005, Le conflit des héritages. Paris: Du Théâtre, H-S no.16.

Van Den Dries, L. (2005). Corpus Jan Fabre, observations sur un processus de création. Paris: L’Arche.

Publisher's Note Springer Nature remains neutral with regard to jurisdictional claims in published maps and institutional affiliations. 